\title{
Skin Care Formulations and Lipid Carriers as Skin Moisturizing Agents
}

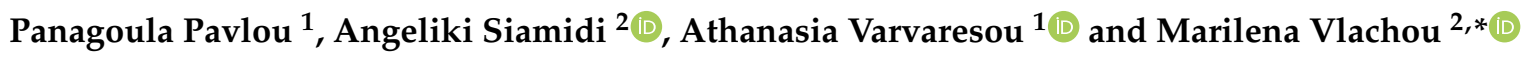 \\ 1 Laboratory of Chemistry-Biochemistry-Cosmetic Science, Section of Aesthetics and Cosmetic Science, \\ Department of Biomedical Sciences, School of Health and Care Sciences, University of West Attica, \\ 28 Ag. Spyridonos Str., Panepistimioupolis Egaleo Park, 12243 Athens, Greece; ppavlou@uniwa.gr (P.P.); \\ avarvares@uniwa.gr (A.V.) \\ 2 Section of Pharmaceutical Technology, Department of Pharmacy, School of Health Sciences, National and \\ Kapodistrian University of Athens, 15784 Athens, Greece; asiamidi@pharm.uoa.gr \\ * Correspondence: vlachou@pharm.uoa.gr; Tel.: +30-21-0727-4674
}

Citation: Pavlou, P.; Siamidi, A.; Varvaresou, A.; Vlachou, M. Skin Care Formulations and Lipid Carriers as Skin Moisturizing Agents. Cosmetics 2021, 8, 89. https:// doi.org/10.3390/cosmetics8030089

Academic Editors: Sophia Letsiou and Sophia Hatziantoniou

Received: 17 August 2021

Accepted: 15 September 2021

Published: 17 September 2021

Publisher's Note: MDPI stays neutral with regard to jurisdictional claims in published maps and institutional affiliations.

Copyright: (c) 2021 by the authors. Licensee MDPI, Basel, Switzerland. This article is an open access article distributed under the terms and conditions of the Creative Commons Attribution (CC BY) license (https:/ / creativecommons.org/licenses/by/ $4.0 /)$.

\begin{abstract}
Skin care formulations have evolved as the interaction of health and beauty products for the skin. Their benefits are based on the combination of cosmetic active ingredients and targeted application. Cosmetic actives have been used in novel formulations for decades (sunscreens, anti-aging treatments, etc.), but the problems with their low solubility, low penetration, and physicochemical instability when applied to the skin have yet to be solved. One way to circumvent these shortcomings is to use lipid carriers, which are known to play an important role in the solubility of poorly soluble compounds by facilitating skin permeation and improving stability. This review addresses recent advances in skin care products that use novel nanotechnology-based lipid systems (liposomes, solid lipid nanoparticles, etc.) to deliver moisturizing cosmetic actives and improve product efficacy.
\end{abstract}

Keywords: cosmetic active ingredients; hydration; nano skin care formulations; nanocarriers; occlusion; stratum corneum; lipid vehicles; emollients; cosmetics; skin penetration

\section{Introduction}

The selectively permeable outer layer of the epidermis, the stratum corneum (SC), protects the body from dehydration and other environmental stresses. There are three possible pathways for drug penetration through the SC: the intracellular, the shunt (via sweat glands and hair follicles), and the intercellular pathway. As shown in Figure 1, the intracellular pathway appears to be the shortest, but unfortunately, it requires passage through closely associated corneocytes and intercellular lipid layers, resulting in slower penetration of hydrophobic active pharmaceutical ingredients (APIs). On the other hand, the shunt pathway, previously thought to be a minor contributor to drug absorption (as it represents only $0.1 \%$ of total human skin), was recently found to be a potentially remarkable pathway for a variety of APIs (i.e., charged molecules, nanomedicines, etc.). Therefore, most topically applied agents penetrate SC through the intercellular pathway (lipid layers). By altering the intercellular lipid composition of the SC, water diffusion can be significantly increased [1].

Skin care products contain active ingredients that are either hydrophilic or hydrophobic; each category has its limitations when used in topical formulations. To overcome these limitations, many factors should be considered, such as the skin area in the human body, the duration of application, the mechanism of action, and the potential side effects. For example, hydrophilic agents cannot penetrate the SC due to their polarity, while hydrophobic agents have an unpleasant oily texture for patients [3]. The use of transdermal delivery systems (TDDS) is an alternative technique that facilitates the penetration of active ingredients through the skin. Like other topical formulations, these systems offer non-invasive drug delivery compared to parenteral routes, but have limitations due to the 
low permeability of drugs through the skin barrier. Therefore, the development of a robust TDDS is required to improve drug delivery [3].

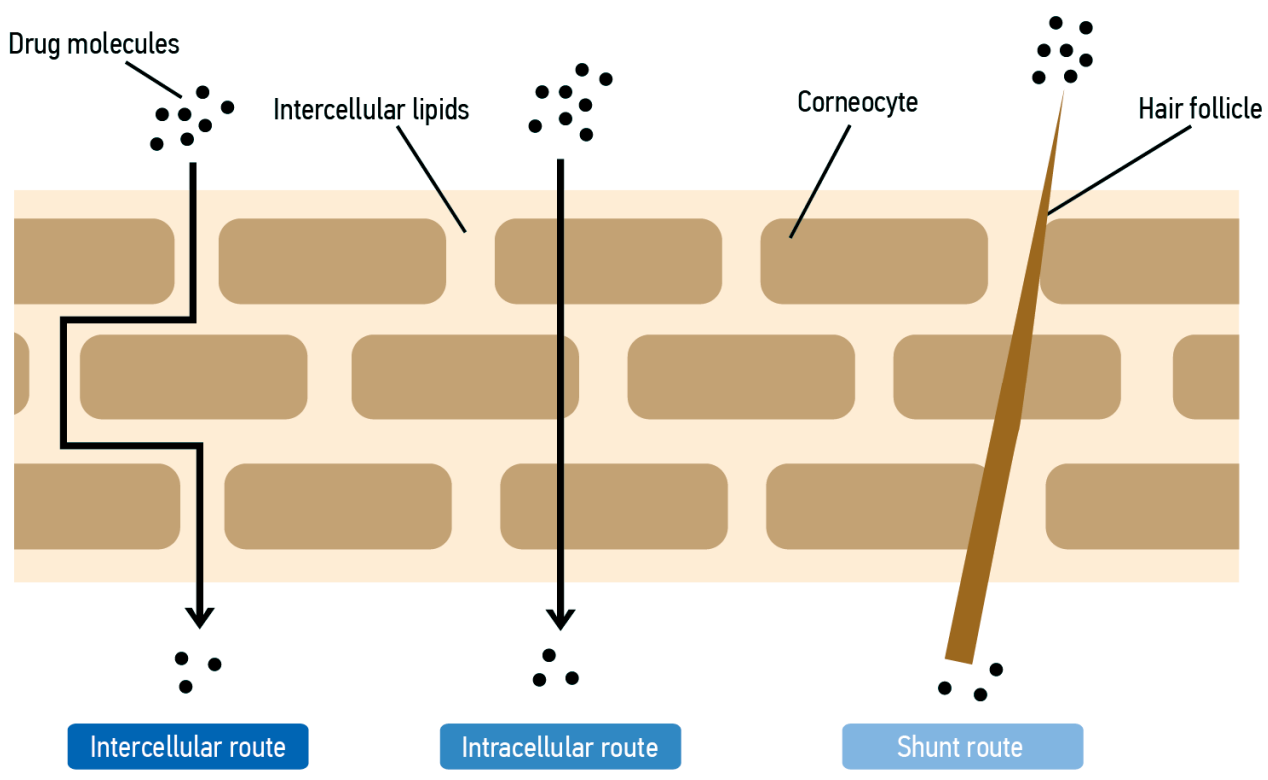

Figure 1. Routes of drug penetration through the SC [2].

\section{Skin Care Products-Cosmeceuticals}

The term "cosmeceutical" was first mentioned in 1984 by Dr. Albert Kligman (University of Pennsylvania), who used it to describe a new category of products that could be classified as more than just cosmetics or pharmaceuticals. According to Dr. Kligman, a cosmeceutical is "a topical preparation that is sold as a cosmetic but has performance characteristics suggestive of a pharmaceutical effect" and therefore might have a pharmaceutical therapeutic effect but not essentially a biological therapeutic benefit. Since the discovery of the anti-ageing effects of tretinoin, the use of alpha hydroxy acids as exfoliants and for skin rejuvenation, and the use of vitamin $C$ in topical formulations as an antioxidant, the market for cosmeceuticals has grown exponentially and has taken over the personal care industry. Since the term "cosmeceutical" is not yet well established, it is often used in the cosmetic industry to emphasise the specific mechanism of action of the ingredients included in modern skin care formulations. Despite the confusion about their definition and scope, about $40 \%$ of dermatologist prescriptions around the world are cosmeceuticals [4]. Nowadays, the use of nanotechnology to improve the efficacy of APIS is a great success [5].

\section{Skin Hydration}

To maintain optimal moisture levels in the skin, many factors must be considered: The intercellular lipids form a barrier to the passage of water through the tissue; the diffusion pathway, which consists of SC layers and many corneocytes, is long and delays water loss; and the natural moisturizer, which is a complex mixture of low-molecular-weight, water-soluble compounds, is first formed within the corneocytes [6].

Moisturizers are a very important class of cosmetics because they are widely used not only for their preventive effects (xeroderma, preventing the appearance of dryness and delaying premature skin aging), but also because they help in dermatological therapies for various skin diseases and provide a healthy appearance [7]. The evaluation of moisturizers is about assessing their ability to improve these important functions of the skin.

The purpose of formulations containing moisturizing agents is to ensure an optimal balance between water and skin lipids by ensuring the water content in the skin in various ways [8]: 
1. by the addition of humectants (glycerol, sorbitol, sodium hyaluronate with low MW, elements of natural hydration factors). These are hygroscopic substances that increase the ability of the SC cells to retain water.

2. by adding film-forming/occlusive agents or emollients [hydrocarbons (petrolatum, paraffin oil, perhydrosqualene), silicone oils, animal or vegetable oils, fatty alcohols, especially stearyl and cetyl, waxes (from shea, bees)]. Many of the lipophilic moisturizers act by forming a nearly impermeable layer on the surface of the SC, thereby reducing water evaporation from the SC. Such moisturizers are called occlusive moisturizers and do not normally penetrate the SC. However, some moisturizers appear to increase the water content in the SC without forming an impermeable layer on the surface of the SC. The mode of action of such non-occlusive lipophilic moisturizers is as yet unclear [9].

3. by the addition of lipids which mimic the lipids in SC (ceramides).

This review attempts to summarize the novel lipid carriers and moisturizing factors distributed by lipid vehicles currently used in skin care formulations.

\section{Novel Nanocarriers for Skin Care Products}

\subsection{Liposomes}

Liposomes are spherical vesicles composed of a bilayer of phospholipids. Their similarity to the membranes of somatic cells has made them one of the most commonly used drug delivery systems. The inner core and lipid membranes can accommodate both hydrophilic and hydrophobic drugs. However, liposomes have a low stability which can lead to rapid leakage [3].

To overcome this limitation, it has been proposed to modify the surface of liposomes by polymer coating or cell-penetrating peptide or by conjugating the surface with polyethylene glycol. Indeed, researchers have used layer-by-layer technology based on the electrostatic interaction of polyelectrolytes to improve the stability of transdermal drug delivery systems. More specifically, dihexadecyl phosphate was used to add a negative charge to liposomes, and then multilayered liposomes were developed (with $\leq 10$ alternating layers of cationic chitosan followed by anionic sodium hyaluronate) using the above technique. The resulting particles (size $\approx 530 \mathrm{~nm}$ ) exhibited an alternative change in zeta potential. Using differential scanning calorimetry and transmission electron microscopy, the multilayered liposomes appeared to form a spherical polyelectrolyte complex after deposition. Moreover, observations of the size distribution after 1 week of formation showed that the particles coated with even layers of polyelectrolytes, hyaluronate and chitosan, were more stable than those coated with odd layers. When surfactant Triton X-100 was used, the number of bilayers increased and the stability of the membrane was increased. The layer-by-layer technique also changed the drug release in a sustained manner. The in vitro skin permeation study showed that the loaded multilayer liposomes exhibited similar skin permeability, which was superior to the uncoated liposomes. Therefore, the properly coated multilayer liposomes with sodium hyaluronate as humectant and chitosan may improve stability and serve as a potential transdermal drug delivery system [3].

Modified liposomes (transfersomes and ethosomes) are also currently used for transdermal drug delivery. Ethosomes, first described by Touitou et al. [10], are lipid-based carriers characterized by a higher ethanol content compared to liposomes. Ethanol can break the lipid bilayer of the skin and makes the lipid membrane loose, allowing the vesicle to penetrate into the SC and better distribute the drug in the lipids of SC. For all these reasons, ethosomes show better penetration through the $\mathrm{SC}$ and have a significantly higher transdermal flux [11].

\subsection{Niosomes}

Niosomes are vesicles formed by non-ionic surfactants and cholesterol incorporation. They are biodegradable, relatively nontoxic, and inexpensive. Since they have a bilayer, they are structurally similar, but the materials used to make them make them 
more stable [12]. Niosomes can be classified into different groups according to their size (small/large unilamellar vesicles) or the number of lamellar layers (multilamellar/small unilamellar vesicles). Their size is also a determining factor affecting the choice of route of administration. Vesicles with a size in the submicron range are suitable for intravenous or transdermal administration, whereas vesicles with a size of up to $10 \mu \mathrm{m}$ are commonly used for per os, intramuscular and nasal administration. Small unilamellar vesicles (size from 10 to $100 \mathrm{~nm}$ ) are prepared from multilamellar vesicles by methods such as sonication, extrusion under high pressure and homogenization under high shear. Compared to other types of niosomes, they are thermodynamically less stable and have poor loading capacity for hydrophilic drugs and a higher tendency to form aggregates. Large unilamellar vesicles (0.1-1 $\mu \mathrm{m}$ diameter) consist of a single bilayer surrounding the aqueous core and can be used for encapsulation of hydrophilic drug molecules. Multilamellar vesicles ( $0.5 \mu \mathrm{m}$ to $10 \mu \mathrm{m}$ diameter) consist of multiple bilayers surrounding the aqueous lipid compartments separately. They can be easily prepared without complicated techniques and are more stable compared to the other two types of niosomes. Moreover, they can easily accommodate lipophilic drugs due to the multilayered membranes. [13].

\subsection{Nanostructured Lipid Carriers and Solid Lipid Nanoparticles}

Solid lipid nanoparticles (SLN) and nanostructured lipid carriers (NLC) are lipid nanoparticles that have been thoroughly studied for application to the skin. SLNs are prepared by replacing the liquid lipid of an oil-in-water emulsion with one or a mixture of solid lipids, while NLCs are prepared using mixtures of solid lipids with liquid lipids (oils), preferably in a ratio of 70:30 to a ratio of 99.9:0.1. The resulting matrix of NLCs has a lower melting point compared to SLNs, but still, both are solid at room/body temperature [14].

Aqueous dispersions of lipid nanoparticles are usually incorporated into a semi-solid dermal carrier (cream or gel) for topical application. These formulations can be prepared by the following methods:

1. mixing SLN/NLC with existing products

2. adding viscosity improvers to the aqueous phase

3. directly preparing a semi-solid formulation containing only nanoparticles in a onestep process.

Hydrogel formulations containing hydroxyethylcellulose 4000, xanthan gum, acrylate polymers and chitosan as gelling agents, also containing SLN and NLC, have already been prepared and analyzed; the results showed good physical stability. The term nanolipid gel refers to a system consisting of lipid nanoparticles incorporated into a gel base. Nanolipid gels can be formulated by adding a gelling agent to the SLN-containing dispersion [15].

\subsubsection{Film Formation and Occlusive Property of NLCs and SLNs}

The SC contains $\approx 10-20 \%$ water. If water evaporation from the skin to the atmosphere increases and the water content falls below this level, the protective layer of the SC may no longer be intact. In this case, topical formulations with occlusive properties must be applied to the SC to regenerate the water content. As a result, the SC will begin to repair itself. Occlusion reduces the evaporation of water from the skin into the atmosphere, leaving the water in the skin. The SC swells, resulting in improved permeation of medications. Unfortunately, many topical preparations that have good occlusive properties (e.g., petrolatum, fats, fatty acids, etc.) do not have a satisfactory cosmetic or aesthetic appearance. For this reason, various emulsions (w/o or $\mathrm{o} / \mathrm{w}$ ) have been developed to provide a middle ground between occlusive performance and skin appearance/texture. Due to their occlusive properties, emulsions are valuable as they moisturise the skin [16].

NLC and SLN can form a film with occlusive properties that prevent water loss by evaporation, increase skin hydration, and thus improve skin appearance, especially in conditions such as eczema. Due to their ultrafine size, NLC and SLN can provide better occlusion compared to macroemulsions [16,17]. The occlusive properties of SLN and NLC formulations due to film formation after application to the skin, which results in less 
water evaporation, have been extensively studied (Figure 2). In particular, researchers have shown that SLN have a higher occlusion factor compared to NLC formulations with the same lipid content. Moreover, when comparing NLC formulations with different oil content, the occlusion factor showed a decrease when the oil content increased [18].

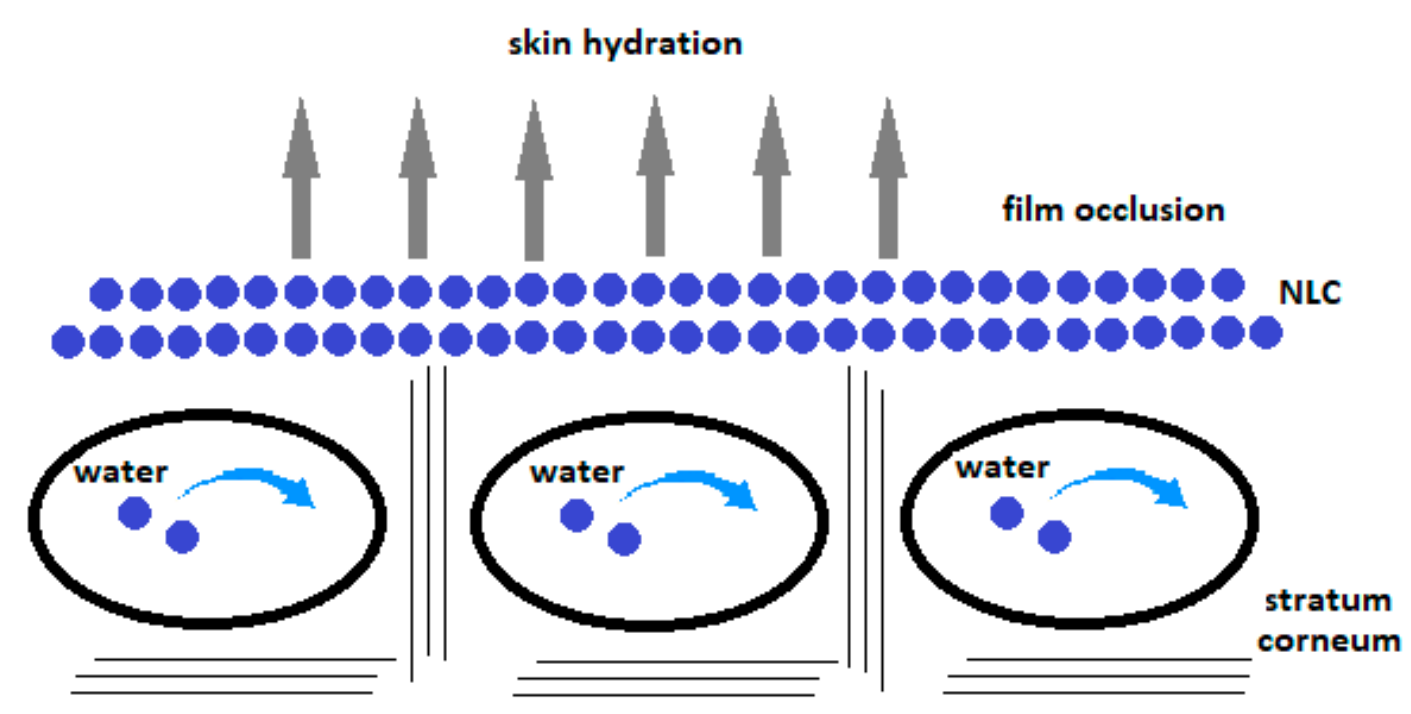

Figure 2. High concentration of lipid (50-60\%) present in NLC formulation act as occlusive agent and is responsible for retaining the moisture in the SC [19].

A group of researchers compared the first NLC-containing cosmetic product to hit the market, Cutanvoa Nanorepair Q10, with a conventional o/w cream without NLCs [20]. The results indicated that Cutanvoa Nanorepair Q10 was more efficient, achieved longterm stability after incorporation into xanthan gum hydrogels, and exhibited higher skin hydration without irritation [21].

4.3.2. The Effects of Varying Lipid Concentrations, Lipid and Oil Ratio, and the Addition of Propylene Glycol and Lecithin on the Long-Term Physical Stability of NLC, Skin Hydration, and TEWL

Researchers prepared NLC formulations, analyzed their particle size, zeta potential, viscosity, and stability, and then recorded the hydration and occlusion of the skin of the forearms of 20 female subjects after application [22]. The results showed that the NLC formulations with the highest lipid concentration, the highest concentration of solid lipids, and additional propylene glycol exhibited the highest physical stability. In particular, propylene glycol contributed to the reduction of particle size, thus improving the long-term physical stability of the formulation. Moreover, the addition of propylene glycol or lecithin helped to improve the occlusive properties of the formulation, as at least a $60 \%$ reduction in TEWL was observed. Therefore, NLCs with high lipid content can be effective cosmeceutics to improve skin hydration [22].

\subsection{Nanoemulsions}

The term nanoemulsion is used to describe a kinetically or thermodynamically stable liquid dispersion containing the oil and water phases in combination with a surfactant. Depending on their composition, there are three different types of nanoemulsions: water in oil, oil in water and bicontinuous. Their dispersed phase (small particles or droplets of 50-200 nm) has a very low interfacial tension between oil and water. Their lipophilic core surrounded by a monomolecular layer of phospholipids makes them more suitable for the delivery of lipophilic drugs. Nanoemulsions do not exhibit sedimentation, coalescence, foaming and flocculation like emulsions of macromolecules. On the contrary, they are transparent or translucent and exhibit properties such as low viscosity, high kinetic stability, 
large interfacial area and high solubilization capacity. In skin care formulations, they can provide rapid skin penetration, active ingredient transport and hydration [23,24].

\subsection{Gold Nanoparticles}

Gold nanoparticles or nanogold (5-400 $\mathrm{nm}$ in diameter) are extremely stable, biocompatible, and noncytotoxic particles. Their interparticle interactions and assembly play an important role in determining their properties. Their shape (nanosphere, nanoshell, nanocluster, nanorod, nanostar, nanocube, branched and nanotriangles), size, dielectric properties and environmental conditions have a strong influence on their resonance frequency. Their color ranges from red to violet/blue and is almost black due to aggregation. They are stable in liquid or dried form and do not bleach after staining on membranes. Gold nanoparticles are considered beneficial in formulations due to their high drug loading capacity and easy penetration into the target cell due to their small size and large surface area, shape and crystallinity, but also due to their properties, i.e., acceleration of blood circulation, anti-inflammatory and antiseptic effect, improvement of skin firmness and elasticity, retardation of the aging process and vitalization of skin metabolism [23].

\subsection{Nanospheres}

Nanospheres (10 to $200 \mathrm{~nm}$ in diameter) are spherical particles with core-shell structure. They can be crystalline or amorphous. Nanospheres can be divided into two categories: biodegradable (gelatin, modified starch and albumin) and non-biodegradable (polylactic acid). The active ingredient can be entrapped, dissolved, attached, or encapsulated within the polymer's matrix system, protecting it from chemical and enzymatic degradation. As drug delivery systems, they have great potential as they can entrap poorly soluble or absorbed labile biological agents, enzymes and genes. In cosmetics, they are used to deliver active ingredients into the deeper layers of the skin so that they can exert their effects more precisely and efficiently at the affected site. They play an important role in protecting against actinic aging and are mainly used in moisturizers, anti-wrinkle and anti-acne creams [23].

Many nano-formulations are commercially available [5,25]; Table 1 summarizes those with hydration currently on the market.

Table 1. Commercially available nanocosmeceutical formulations with hydration use.

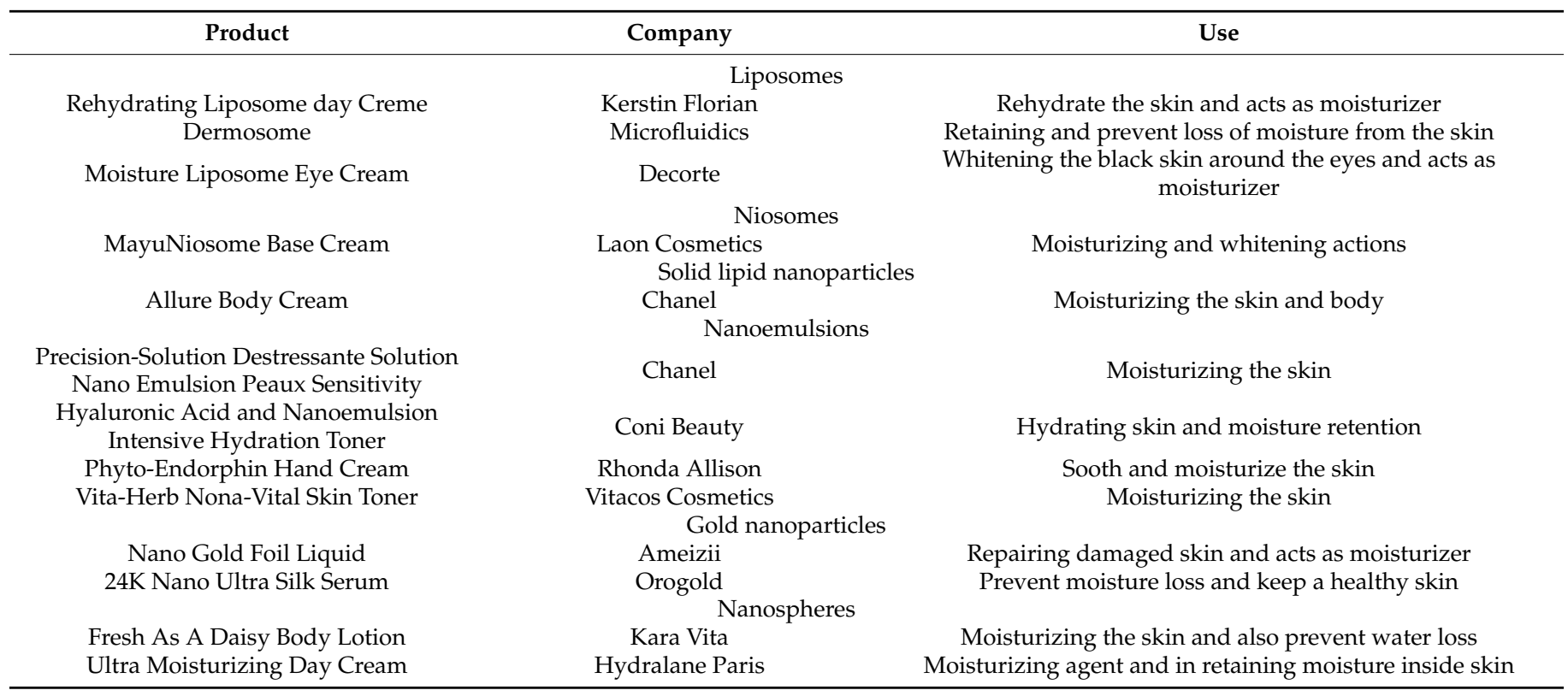




\section{Recent Studies on Moisturizing Factors Distributed through Lipid Vehicles 5.1. Squalene}

Squalene is widely distributed in nature (olive, palm, wheat germ, amaranth and rice bran oils), but its richest source is shark liver oil. It is an intermediate in cholesterol biosynthesis and is considered the main component of polyunsaturated lipids in SC. It is used as an emollient, antioxidant, moisturizer and for its antitumor activities, and also as an excipient in lipid emulsions and NLCs. Researchers prepared O/W lipid emulsions with 18 different natural oils in order to study the effects on the particle size and stability of the emulsion. The results showed that squalene could form the most stable emulsions. Another important finding was that when a lipophilic active agent was introduced into the discontinuous oil phase, the in vitro release from squalene emulsion was the slowest one [26].

A group of scientists found that the lipophilicity of NLCs (mean particle size of $200 \mathrm{~nm}$ ) composed of Precirol ${ }^{\circledR}$ and squalene decreased with an increase in the amount of squalene in the formulations [27]. 8-Methoxypsoralen (a psoralen derivative) was incorporated into NLCs with squalene to investigate their ability to permeate through the skin for the treatment of psoriasis. The results showed improved permeation and altered drug release. In particular, when the NLCs were stabilized with Tween ${ }^{\circledR} 80$, the drug flux was 2.8 times higher than with a conventional emulsion [28].

\subsection{Phyto-Based Nano-Formulations}

Phytobioactive compounds (catechins, gallic acids, epicatechins, curcumin, hy-droxylbenzoic acid and cinnamic acid, quercetin, ascorbic acid, luteolin, alpha- and beta-carotene, complex polysaccharides, and fatty acids) are used in cosmetic formulations not only for their dermatocosmetic effects (moisturizing, anti-aging, sun protection), but also because they can enhance antibacterial, antifungal, anticarcinogenic and anti-inflammatory biological effects. Several macroscopic active phytoin ingredients are currently used in cosmetic products. Unfortunately, there are limitations to their use depending on their solubility and size. These include low skin penetration, low stability and low whitening effect. For these reasons, researchers are looking for new technologies to improve their effect in cosmetic products [29]. The use of nanotechnology (nanoemulsions, nanoliposomes, and SLN) has solved most of these problems and allowed the production of effective moisturizers containing phytobioactive substances. Currently, many phytobased nano-moisturizing systems are being developed. Researchers have formulated SLN with safranol (diameter $100 \mathrm{~nm}$ ) to enhance the moisturizing and UV-protective effects [30]. In addition, scientists have developed rice bran oil nanoemulsions that have moisturizing effects in atopic dermatitis and psoriasis [31]. Another group of researchers formulated nanoemulsions (92-233 nm particle size) from Opuntia ficus-indica (L.) extract [32]. Therefore, plant oils derived from various nanoscale phytocompounds and administered with nanotechnologies will be the future of nanocosmeceuticals to enhance moisturizing effects [29].

\subsection{Argan/Jojoba Oil Nanostructured Lipid Carriers}

Argan oil is a natural oil used in both dermatological and cosmetic formulations because it moisturises the skin, has antioxidant properties, inhibits ageing, and provides protection. It contains relatively high amounts of squalene, $\gamma$-tocopherol and unsaturated fatty acids (oleic acid and linoleic acid). Scientists have formulated a preparation of NLC systems with argan oil as a liquid lipid to improve skin hydration [33]. In vivo evaluation was performed on volunteers by measuring various skin surface parameters over a period of 1 month and showed improvement in skin hydration. According to the results of this study, the NLC systems with argan oil could have a synergistic effect on skin hydration as the NLCs occlude and the argan oil moisturises. This NCL system could be improved by adding an additional anti-ageing compound [33].

Another group of researchers had also formulated semi-solid NLC products using argan or jojoba oil as liquid lipids and carbomer (Carbopol ${ }^{\circledR} 934$ or 980$)$ as gelling agent [33]. Since jojoba oil has structural similarities to human skin oil (sebum), applying this oil to the 
skin may send a biological message that the skin has produced enough sebum so that oil production can be balanced. It is composed of oleic acid (14\%), eicosenoic acid (70\%) and docosenoic acid (16\%). To prepare the formulations, the lipid phase (glyceryl behenate and argan or jojoba oil) was heated $5-10^{\circ} \mathrm{C}$ above the melting point and added to the aqueous phase (Tween ${ }^{\circledR} 80$, cetrimide, water and glycerol), which was previously heated to the same temperature with stirring. Then a sonication probe was used to break up the lipid droplets and the carbomer was added. The results of instrumental analysis and sensory and in vivo efficacy evaluation showed increased hydration due to the formation of an occlusive film on SC. This effect was more pronounced when smaller NLCs with a low solid lipid content were used [34].

\subsection{Macro-Sized Particles, Made of a Hydrogel Polymer, Loaded with Olive Oil}

An attractive alternative to NLC systems loaded with exotic oils could be alginate beads loaded with olive oil. In this way, the new sunscreens and moisturizing formulations would contain a natural product and a biocompatible polymer. Olive oil contains squalene and vitamin E, which are useful ingredients in skin care formulations. As mentioned earlier, squalene moisturizes and protects the skin, while vitamin E increases skin hydration and water-binding capacity [35]. On the other hand, alginate as a hydrogel with suitable mechanical and biocompatible properties can moisturize the skin, play an important role in the wound healing process, and deliver active ingredients to the SC [36,37].

Researchers have formulated alginate beads encapsulated in olive oil and incorporated them into semi-solid vehicles to produce cosmetic products that can be used in the skin of the elderly as moisturizers and photoprotectants. The results of this study show that the type of olive oil used determines the antioxidant activity and polyphenol content. The in vitro results also indicate protection against UV rays, while the in vivo results indicate a moisturizing effect [38].

\subsection{Silver-Nanolipid Complex with Hemp Seed Oil for Application to Atopic Dermatitis Skin}

Atopic dermatitis is a condition characterized by damaged SC and by the presence of bacteria that are not part of the usual bacterial flora. Mild conditions require frequent application of moisturizers, while moderate to severe conditions require the use of pharmaceutical creams (e.g., corticosteroids or calcineurin inhibitors). Since the required long-term drug treatment can lead to atrophy of the skin, researchers have succeeded in formulating a nanocosmeceutical with improved skin care properties without active ingredients for the treatment of mild to moderate atopic dermatitis. This was achieved by using NLCs and antimicrobial silver. Specifically, the NLCs were prepared using cetyl palmitate, Tegocare 450, Inutec SP1 and hemp seed oil. It is known that hemp seed oil contains more than $60 \%$ of polyunsaturated $\alpha$-linolenic acid, a major component of the hydrolipid layer. Since $\alpha$-linolenic acid is sensitive to oxidative stress, hempseed oil was incorporated into the matrix of the NLC to protect it from degradation by oxidative species [39].

\subsection{Exotic Vegetable Oils for Cosmetic o/w Nanoemulsions}

Lanolin is a substance composed of a mixture of naturally formed esters of higher alcohols and fatty acids [40] and is widely used in pharmaceuticals and cosmetics as a carrier for drugs and cosmetics due to its beneficial properties (e.g., low toxicity, occlusive ability, skin moisturizing). Recently, a group of researchers studied the influence of lanolin derivatives (ethoxylated and acetylated lanolin) on the physicochemical properties and stability of vegetable oil-based nanoemulsions. Specifically, raspberry, passion fruit and peach oils (1:1:1) were used in combination with lanolin derivatives to form nanoemulsions that increased skin hydration and lipid content without altering skin pH. Although it was found that those containing ethoxylated lanolin were more stable than those with acetylated lanolin, this project proved that nanoemulsions based on exotic vegetable oils and lanolin derivatives can be used as carriers for cosmetic products [41,42]. 


\subsection{Silicone Vesicles and Matrices}

Silicones can serve as transport vesicles for active ingredients through the skin by physical association. The vesicles required for assembly (nanomicron- to submicronsized vesicular structures made of silicone polyethers) are very stable in aqueous media, making them ideal materials for personal care products. Active ingredients that can be delivered through these vesicles include skin care ingredients (vitamins A and E, lanolin oil), humectants (lanolin alcohol, cetearyl octanoate, sodium stearoyl lactylate), colorants, and emollients (mineral/jojoba oil and polydimethylsiloxane) [43].

Common silicone oils (e.g., dimethicone) are used in cosmetics due to their high permeability, which makes them ideal for modified release formulations. On the other hand, crosslinked silicones (e.g., elastomers and adhesives) are relatively new compounds used in cosmetics. In particular, the linkages between the polymer chains make the silicone elastomers a solid material in which the active ingredient can be entrapped. The formation of these systems is based on the condensation of a silica derivative with hy-droxyl terminated silicone polymers, on mixing the active ingredient with the silicone polymers before cross-linking, or on swelling the cross-linked silicone matrix with a suitable solvent and using this solvent to carry the active ingredient into the matrix. Generally, the release rate can be increased if the active agent is combined with a silicone surfactant [43].

\subsection{Hyaluronic Acid}

Hyaluronic acid (HA) is one of the most important components of the extracellular matrix in the epidermis and dermis, has moisturizing and skin-repairing properties. Unfortunately, it cannot penetrate deep skin due to its large molecular weight (200-325 kDa). A group of scientists developed an ethosomal delivery system (diameter $110 \mathrm{~nm}$ ) that encapsulates HA with a skin-penetrating peptide (SPACE) conjugated to phospholipids to improve its topical delivery [44]. The system enhanced HA penetration into the epidermis and dermis, disrupting SC. It was found that the HA concentrations in the skin were about 1000 times higher than those in the blood.

The researchers formulated an alcohol-free $\mathrm{O} / \mathrm{W}$ nanoemulsion $\mathrm{HA}$ that penetrated through the SC and diffused deeper into the dermis via the shunt and intercellular pathways. The penetration mechanism might be closely related to the transmembrane concentration gradient, carrier properties and penetration enhancers [45].

\subsection{Ceramides}

Ceramide is one of the major intercellular lipid matrices of the SC, which binds to proteins on the cell surface via ester bonds and forms a barrier in the $\mathrm{SC}$, preventing water loss and protecting it from external stimuli [46]. Being a highly crystalline substance, it is not so easy to use in cosmetics due to its low solubility, poor water dispersion and easy crystallization. Despite this fact, researchers have formulated a positively charged nanoemulsion with ceramides that has improved stability and solubility [47].

\section{Conclusions/Future Perspectives}

Skin care products integrated with nanotechnology are spreading rapidly around the world and are proving to be particularly important in the treatment of various skin conditions. To date, there are no specific guidelines, so these products are spreading rapidly in the personal care market. However, there are concerns regarding the environment and side effects that should be taken care of. There is therefore an urgent need for harmonized guidelines and regulations for the use of nanomaterials.

Author Contributions: Conceptualization, M.V.; methodology, P.P.; investigation, P.P. and A.S.; data curation, A.S.; writing-original draft preparation, P.P. and A.S.; writing-review and editing, M.V. and A.V.; supervision, M.V.; project administration, M.V. All authors have read and agreed to the published version of the manuscript.

Funding: This research received no external funding. 
Conflicts of Interest: The authors declare no conflict of interest.

\section{References}

1. Kalia, Y.N.; Guy, R.H. Modeling transdermal drug release. Adv. Drug Deliv. Rev. 2001, 48, 159-172. [CrossRef]

2. Czajkowska-Kosnik, A.; Szekalska, M.; Winnicka, K. Nanostructured lipid carriers: A potential use for skin drug delivery systems. Pharmacol. Rep. 2019, 71, 156-166. [CrossRef]

3. Jeon, S.; Yoo, C.Y.; Park, S.N. Improved stability and skin permeability of sodium hyaluronate-chitosan multilayered liposomes by Layer-by-Layer electrostatic deposition for quercetin delivery. Colloids Surf. B Biointerfaces 2015, 129, 7-14. [CrossRef] [PubMed]

4. Pandey, A.; Jatana, G.K.; Sonthalia, S. Cosmeceuticals. In Stat Pearls; StatPearls Publishing: Treasure Island, FL, USA, 2021. Available online: https:/ / www.ncbi.nlm.nih.gov/books/NBK544223/ (accessed on 1 August 2021).

5. Dhapte-Pawar, V.; Kadam, S.; Saptarsi, S.; Kenjale, P.P. Nanocosmeceuticals: Facets and aspects. Future Sci. OA 2020 , 6, FSO613. [CrossRef] [PubMed]

6. Rawlings, A.V.; Harding, C.R. Moisturization and skin barrier function. Dermatol. Ther. 2004, 17, 43-48. [CrossRef] [PubMed]

7. Loden, M. The clinical benefit of moisturizers. J. Eur. Acad. Dermatol. Venereol. 2005, 19, 672-688. [CrossRef]

8. Duplan, H.; Nocera, T. Skin hydration and hydrating products. Ann. Dermatol. Venereol. 2018, 145, 376-384. [CrossRef] [PubMed]

9. Caussin, J.; Gooris, G.S.; Bouwstra, J.A. FTIR studies show lipophilic moisturizers to interact with stratum corneum lipids, rendering the more densely packed. Biochim. Biophys. Acta 2008, 1778, 1517-1524. [CrossRef] [PubMed]

10. Touitou, E.; Dayan, N.; Bergelson, L.; Godin, B.; Eliaz, M. Ethosomes-novel vesicular carriers for enhanced delivery: Characterization and skin penetration properties. J. Control. Release 2000, 65, 403-418. [CrossRef]

11. Gollavilli, H.; Hegde, A.R.; Managuli, R.S.; Bhaskar, K.V.; Dengale, S.J.; Reddy, M.S.; Kalthur, G.; Mutalik, S. Naringin nanoethosomal novel sunscreen creams: Development and performance evaluation. Colloids Surf. B Biointerfaces 2020, $193,111122$. [CrossRef]

12. Kazi, K.M.; Mandal, A.S.; Biswas, N.; Guha, A.; Chatterjee, S.; Behera, M.; Kuotsu, K. Niosome: A future of targeted drug delivery systems. J. Adv. Pharm. Technol. Res. 2010, 1, 374-380.

13. Chena, S.; Hanninga, S.; Falconerb, J.; Lockea, M.; Wena, J. Recent advances in non-ionic surfactant vesicles (niosomes): Fabrication, characterization, pharmaceutical and cosmetic applications. Eur. J. Pharm. Biopharm. 2019, 144, 18-39. [CrossRef] [PubMed]

14. Aziz, Z.A.A.; Mohd-Nasir, H.; Ahmad, A.; Setapar, S.H.M.; Peng, W.L.; Chuo, S.C.; Khatoon, A.; Umar, K.; Yaqoob, A.A.; Nasir, M.; et al. Role of Nanotechnology for Design and Development of Cosmeceutical: Application in Makeup and Skin Care. Front. Chem. 2019, 7, 739. [CrossRef] [PubMed]

15. Wavikar, P.; Vavia, P. Nanolipidgel for Enhanced Skin Deposition and Improved Antifungal Activity. AAPS PharmSciTech 2013, 14, 222-233. [CrossRef] [PubMed]

16. Müller, R.H.; Petersen, R.D.; Hommoss, A.; Pardeike, J. Nanostructured lipid carriers (NLC) in cosmetic dermal products. Adv. Drug Deliv. Rev. 2007, 59, 522-530. [CrossRef]

17. Patel, D.; Kumar, V.; Kesharwani, R.; Mazumdar, B. Lipid Nanoparticle a Novel Carrier for Cosmetics and Topical Preparation: A Review. Inventi Rapid Cosmeceuticals 2015, 3, 1-6.

18. Souto, E.B.; Wissing, S.A.; Barbosa, C.M.; Muller, R.H. Development of a controlled release formulation based on SLN and NLC for topical clotrimazole delivery. Int. J. Pharm. 2004, 278, 71-77. [CrossRef]

19. Chauhan, I.; Yasir, M.; Verma, M.; Singh, A.P. Nanostructured Lipid Carriers: A Groundbreaking Approach for Transdermal Drug Delivery. Adv. Pharm. Bull. 2020, 10, 150-165. [CrossRef]

20. Pardeike, J.; Schwabe, K.; Müller, R.H. Influence of nanostructured lipid carriers (NLC) on the physical properties of the Cutanova Nanorepair Q10 cream and the in vivo skin hydration effect. Adv. Drug Deliv. Rev. 2010, 396, 166-173. [CrossRef]

21. Khater, D.; Nsairat, H.; Odeh, F.; Saleh, M.; Jaber, A.; Alshaer, W.; Al Bawab, A.; Mubarak, M.S. Design, Preparation, and Characterization of Effective Dermal and Transdermal Lipid Nanoparticles: A Review. Cosmetics 2021, 8, 39. [CrossRef]

22. Loo, C.H.; Basri, M.; Ismail, R.; Lau, H.L.N.; Tejo, B.A.; Kanthimathi, M.S.; Hassan, H.A.; Choo, Y.M. Effect of compositions in nanostructured lipid carriers (NLC) on skin hydration and occlusion. Int. J. Nanomed. 2013, 8, 13-22. [CrossRef] [PubMed]

23. Kaul, S.; Gulati, N.; Verma, D.; Mukherjee, S.; Nagaich, U. Role of Nanotechnology in Cosmeceuticals: A Review of Recent Advances. Hindawi J. Pharm. 2018, 2018, 3420204. [CrossRef] [PubMed]

24. Ashaolu, T.J. Environ Nanoemulsions for health, food, and cosmetics: A review. Chem. Lett. 2021, 15, 1-15.

25. Zhou, H.; Luo, D.; Chen, D.; Tan, X.; Bai, X.; Liu, Z.; Yang, X.; Liu, W. Current Advances of Nanocarrier Technology-Based Active Cosmetic Ingredients for Beauty Applications. Clin. Cosmet. Investig. Dermatol. 2021, 14, 867-887. [CrossRef]

26. Chung, H.; Kim, T.W.; Kwon, M.; Kwon, I.C.; Jeong, S.Y. Oil components modulate physical characteristics and function of the natural oil emulsions as drug or gene delivery system. J. Control. Release 2001, 71, 339-350. [CrossRef]

27. Fang, J.Y.; Fang, C.L.; Liu, C.H.; Su, Y.H. Lipid nanoparticles as vehicles for topical psoralen delivery: Solid lipid nanoparticles (SLN) versus nanostructured lipid carriers (NLC). Eur. J. Pharm. Biopharm. 2008, 70, 633-640. [CrossRef] [PubMed]

28. Huang, Z.R.; Lin, Y.K.; Fang, J.Y. Biological and pharmacological activities of squalene and related compounds: Potential uses in cosmetic dermatology. Molecules 2009, 23, 540-554. [CrossRef]

29. Ganesan, P.; Choi, D.K. Current application of phytocompound-based nanocosmeceuticals for beauty and skin therapy. Int. J. Nanomed. 2016, 11, 1987-2007. [CrossRef] 
30. Khameneh, B.; Halimi, V.; Jaafari, M.R.; Golmohammadzadeh, S. Safranal-loaded solid lipid nanoparticles: Evaluation of sunscreen and moisturizing potential for topical applications. Iran. J. Basic Med. Sci. 2015, 18, 58-63.

31. Bernardi, D.S.; Pereira, T.A.; Maciel, N.R.; Bortoloto, J.; Viera, G.S.; Oliveira, G.C.; Rocha-Filho, P.A. Formation and stability of oil-in-water nanoemulsions containing rice bran oil: In vitro and in vivo assessments. J. Nanobiotechnol. 2011, 9, 44. [CrossRef]

32. Ribeiro, R.C.; Barreto, S.M.; Ostrosky, E.A.; da Rocha-Filho, P.A.; Veríssimo, L.M.; Ferrari, M. Production and characterization of cosmetic nanoemulsions containing Opuntia ficus-indica (L.) mill extract as moisturizing agent. Molecules 2015, 20, 2492-2509. [CrossRef]

33. Tichota, D.M.; Silva, A.C.; Lobo, J.M.S.; Amara, M.H. Design, characterization, and clinical evaluation of argan oil nanostructured lipid carriers to improve skin hydration. Int. J. Nanomed. 2014, 9, 3855-3864.

34. Estanqueiro, M.; Conceição, J.; Amaral, M.H.; Sousa Lobo, J.M. Characterization, sensorial evaluation and moisturizing efficacy of nanolipidgel formulations. Int. J. Cosmet Sci. 2014, 36, 159-166. [CrossRef]

35. Korać, R.R.; Khambholja, K.M. Potential of herbs in skin protection from ultraviolet radiation. Pharmacogn. Rev. 2011, 5, 164-173. [CrossRef]

36. Lee, K.Y.; Mooney, D.J. Alginate: Properties and biomedical applications. Prog. Polym. Sci. 2012, 37, 106-126. [CrossRef] [PubMed]

37. Schmitt, A.; Rödel, P.; Anamur, C.; Seeliger, C.; Imhoff, A.B.; Herbst, E.; Vogt, S.; van Griensven, M.; Winter, G.; Engert, J. Calcium Alginate Gels as Stem Cell Matrix-Making Paracrine Stem Cell Activity Available for Enhanced Healing after Surgery. PLoS ONE 2015, 10, e0118937. [CrossRef]

38. Mota, A.H.; Silva, C.O.; Nicolai, M.; Baby, A.; Palma, L.; Rijo, P.; Ascensão, L.; Reis, C.P. Design and evaluation of novel topical formulation with olive oil as natural functional active. Pharm. Dev. Technol. 2017, 23, 794-805. [CrossRef] [PubMed]

39. Keck, M.; Schwabe, K. Silver-Nanolipid Complex for Application to Atopic Dermatitis Skin: Rheological Characterization, In Vivo Efficiency and Theory of Action Cornelia. J. Biomed. Nanotechnol. 2009, 5, 428-436. [CrossRef]

40. Schlossman, M.L.; McCarthy, J.P. Lanolin and its derivatives. J. Amer. Oil Chem. Soc. 1978, 55, 447-450. [CrossRef]

41. Pereira, T.A.; Guerreiro, C.M.; Maruno, M.; Ferrari, M.; Rocha-Filho, P.A. Exotic Vegetable Oils for Cosmetic O/W Nanoemulsions: In Vivo Evaluation. Molecules 2016, 21, 248. [CrossRef] [PubMed]

42. Dini, I.; Laneri, S. The New Challenge of Green Cosmetics: Natural Food Ingredients for Cosmetic Formulations. Molecules 2021, 26, 3921. [CrossRef] [PubMed]

43. Patravale, V.B.; Mandawgade, S.D. Novel cosmetic delivery systems: An application update. Int. J. Cosmet Sci. 2008, 30, 19-33. [CrossRef] [PubMed]

44. Chen, M.; Gupta, V.; Anselmo, A.C.; Muraski, J.A.; Mitragotri, S. Topical delivery of hyaluronic acid into skin using SPACE-peptide carriers. J. Control. Release 2014, 173, 67-74. [CrossRef] [PubMed]

45. Ming, K.; Chen, X.G.; Kweon, D.K.; Park, H.J. Investigations on skin permeation of hyaluronic acid based nanoemulsion as transdermal carrier. Carbohydr. Polym. 2011, 86, 837-843.

46. Uche, L.E.; Gooris, G.S.; Beddoes, C.M.; Bouwstra, J.A. New insight into phase behavior and permeability of skin lipid models based on sphingosine and phytosphingosine ceramides. Biochim. Biophys. Acta Biomembr. 2019, 1861, 1317-1328. [CrossRef]

47. Yilmaz, E.; Borchert, H.H. Design of a phytosphingosine-containing, positively-charged nanoemulsion as a colloidal carrier system for dermal application of ceramides. Eur. J. Pharm. Biopharm. 2005, 60, 91-98. [CrossRef] 